\title{
Short- and long-term mortality after acute myocardial infarction: comparison of patients with and without diabetes mellitus
}

\author{
H. L. Koek · S. S. Soedamah-Muthu · J. W. P. F. Kardaun · E. Gevers · \\ A. de Bruin · J. B. Reitsma • M. L. Bots · D. E. Grobbee
}

Received: 8 May 2007 / Accepted: 27 September 2007/Published online: 10 October 2007

(C) Springer Science+Business Media B.V. 2007

\begin{abstract}
Aims To compare short- and long-term mortality after a first acute myocardial infarction (AMI) in patients with and without diabetes mellitus. Methods and results A nationwide cohort of 2,018 diabetic and 19,547 nondiabetic patients with a first hospitalized AMI in 1995 was identified through linkage of the national hospital discharge register and the population register. Follow-up for mortality lasted until the end of 2000. At 28 days and 5 years respectively, absolute mortality risks were 18 and $53 \%$ in diabetic men, 12 and $31 \%$ in nondiabetic men, 22 and $58 \%$ in diabetic women, and 19 and $42 \%$ in nondiabetic women. Crude mortality was significantly higher in diabetic patients than in nondiabetic patients in both men (28-day hazard ratio (HR) $1.55 ; 95 \%$ confidence interval (CI) 1.32-1.81, 5-year HR 2.01; 95\% CI 1.84-2.21) and women (28-day HR 1.19; 95\% CI 1.03-1.37, 5-year HR 1.53; 95\% CI 1.40-1.67). After multivariate adjustment, risk differences became nonsignificant at 28 days, but diabetes was still associated with a significantly higher long-term mortality in both men (28-day HR 1.16; 95\% CI
\end{abstract}

H. L. Koek · S. S. Soedamah-Muthu · M. L. Bots $(\bowtie) \cdot$

D. E. Grobbee

Julius Center for Health Sciences and Primary Care, University

Medical Center Utrecht, HPN Str. 06.131, P.O. Box 85.060,

3508, AB, Utrecht, The Netherlands

e-mail: m.l.bots@umcutrecht.nl

J. W. P. F. Kardaun · A. de Bruin

Statistics Netherlands, Voorburg, The Netherlands

E. Gevers

Prismant, Utrecht, The Netherlands

J. B. Reitsma

Department of Clinical Epidemiology and Biostatistics,

Academic Medical Center, Amsterdam, The Netherlands
0.99-1.36, 5-year HR 1.49; 95\% CI 1.36-1.64) and women (28-day HR 1.12; 95\% CI 0.97-1.28, 5-year HR 1.39; $95 \%$ CI 1.27-1.52). The interaction between diabetes mellitus and gender did not reach significance in the analyses. Conclusion Our findings in an unselected cohort covering a complete nation show a significantly higher long-term mortality after a first acute myocardial infarction in diabetic patients. Yet, short-term mortality is not significantly higher in diabetic patients. Risks appear to be equally elevated in men and women.

Keywords Myocardial infarction - Registries · Epidemiology · Prognosis · Survival · Mortality · Diabetes mellitus

\section{Introduction}

It is well established that patients with diabetes mellitus (DM) have a higher short-term mortality after acute myocardial infarction (AMI) than patients without DM. Several recent studies have found that hospital, 28-day and 1-year mortality after AMI was approximately 1.5-2 times higher in diabetic patients [1-3]. As it is known that most patients survive beyond this period, it is important to study the difference in long-term survival after an AMI between diabetic and nondiabetic patients as well. Survival after an AMI may be particularly worsened beyond the poorer prognosis already conferred by the presence of DM itself, as diabetic patients with an AMI may experience more severe coronary heart disease and more complications (left ventricular dysfunction and heart failure, significant ventricular arrhythmias) than nondiabetic patients with an AMI [2, 3]. There are relatively few recent studies that address differences in long-term prognosis after an AMI 
between diabetic and nondiabetic patients with follow-up lasting through the second half of the 1990s [4-8]. Moreover, data largely originate from clinical trials or single centers $[4,5,7]$. These studies reported that DM is independently associated with long-term mortality [4-8].

The aim of the present study was to compare short- and long-term mortality after a first hospitalized AMI in patients with and without DM encompassing the entire country by using data from linked national registers.

\section{Methods}

\section{Design}

The study methods, including enrollment of the study population, have recently been described in detail [9]. In brief, cases of first hospitalized AMI in the Netherlands in 1995 were identified by linkage of the Dutch national hospital discharge register and the Dutch population register. Thus, the study included a total of 21,565 patients hospitalized for a first AMI in 1995. These patients had not been previously hospitalized for AMI from 1991 to 1995. Information about demographic characteristics, medical history and hospital outcomes was obtained from the hospital register and the population register. All general and academic hospitals and most specialty hospitals participate in the hospital register. For each hospital admission a new record is created in the hospital register, including the following information: date of birth, gender, numeric part of postal code, hospital-specific patient identification code, type of hospital, admission date and principal and secondary diagnoses of the admission. The principal diagnosis is determined at discharge and is in retrospect the main reason for admission. The diagnoses are coded using the ninth revision and clinical modification of the International Classification of Diseases (ICD-9-CM) [10]. The population register contains information on all registered persons living in the Netherlands, including date of birth, gender, current address, postal code, nationality, native country (both of registered person and his/her parents), date of death and date of emigration. Patients whose parents were both born in the Netherlands were classified as native Dutch. Patients were classified as having a history of cardiovascular disease or having DM when cardiovascular disease (ICD-9-CM [10] codes 390-459 and subcategories, principal diagnoses) or DM (ICD-9-CM [10] code 250 and subcategories, principal and secondary diagnoses) was registered during previous hospital admissions in 1991-1995 and in case of DM also during the index admission in 1995. Data on mortality of the patients were derived by linking their records with the population register and the cause of death certificates. In the population register, it is recorded when a registered person has died. Linkage of the population register with the cause of death certificates yielded information on primary causes of death.

Data analysis

Survival time was calculated as the time from the initial AMI admission date in 1995 to the date of death from any cause, or a patient was censored at the date of loss to follow-up in the population register ( $4 \%$ of patients, e.g. in case of emigration) or the end of study at December 31 , 2000, whichever came first. Crude short-term (28-day), 1year and long-term (5-year) mortality risks were computed for diabetic and nondiabetic patients. Cox proportional hazard analyses were used to examine the association between DM and overall survival in men and women. DM (yes/no) and other predictors, including age (continuous), previous cardiovascular disease (yes/no) and ethnic origin (native or non-native Dutch) were included in the models. Furthermore, Cox proportional hazards models were used to determine whether gender, age, previous cardiovascular disease and ethnic origin were independently associated with overall survival in patients with DM and patients without DM. Interaction terms were included in the models to assess the interaction of gender with DM, age with DM and age with gender.

\section{Results}

The characteristics of the diabetic and nondiabetic patients with a first AMI are presented in Table 1. Most of the diabetic patients were women $(52 \%)$, whereas most of the nondiabetic patients were men $(69 \%)$. In both diabetic and nondiabetic patients, women were older and had a longer duration of hospital stay than men. Diabetic patients were older and more likely to have previous cardiovascular disease (excluding AMI) compared to nondiabetic patients.

During admission, $18 \%$ of the diabetic patients (men $16 \%$, women $20 \%$ ) and $13 \%$ of the nondiabetic patients (men $11 \%$, women 18\%) died. In both diabetic and nondiabetic patients, AMI was the most frequent cause of death (during hospitalization 71 and 82\%, respectively, and during 5 years of follow-up 39 and $45 \%$, respectively).

Table 2 shows the higher absolute short- and long-term mortality risks for diabetic patients compared to nondiabetic patients in men and women.

Crude short- and long-term mortality was significantly higher in diabetic patients than in nondiabetic patients in both men (28-day hazard ratio (HR) 1.55; $95 \%$ confidence interval (CI) 1.32-1.81, 5-year HR 2.01; 95\% CI 1.842.21) and women (28-day HR 1.19; 95\% CI 1.03-1.37, 
Table 1 Characteristics of first acute myocardial infarction patients with and without diabetes mellitus

\begin{tabular}{|c|c|c|c|c|}
\hline & \multicolumn{2}{|c|}{ Patients with diabetes mellitus } & \multicolumn{2}{|c|}{ Patients without diabetes mellitus } \\
\hline & Men & Women & Men & Women \\
\hline Number of patients & 969 & 1,049 & 13,494 & 6,053 \\
\hline \multicolumn{5}{|l|}{ Age at admission (years) } \\
\hline Mean (standard deviation) & $68.7(11.0)$ & $73.2(10.3)$ & $64.0(12.3)$ & $71.6(12.0)$ \\
\hline Prior admission for CVD (\%) & 31.7 & 31.0 & 16.8 & 16.3 \\
\hline \multicolumn{5}{|l|}{ Type of hospital (\%) } \\
\hline Academic & 6.7 & 6.1 & 6.2 & 5.7 \\
\hline General & 93.3 & 93.9 & 93.8 & 94.3 \\
\hline \multicolumn{5}{|l|}{ Length of stay (days) } \\
\hline Mean (standard deviation) & $11.1(8.5)$ & $12.5(11.8)$ & $9.7(7.4)$ & $10.6(9.7)$ \\
\hline Median & 10.0 & 10.0 & 9.0 & 9.0 \\
\hline $\mathrm{P} 25-\mathrm{P} 75^{\mathrm{a}}$ & $7.0-13.0$ & $6.0-16.0$ & $6.0-12.0$ & $6.0-13.0$ \\
\hline Native ethnic origin (\%) & 87.0 & 88.0 & 90.0 & 90.0 \\
\hline
\end{tabular}

CVD = cardiovascular disease, excluding acute myocardial infarction

a 25 th and 75 th percentile

Table 2 Short- and long-term mortality in patients with a first hospitalized acute myocardial infarction (AMI) in the Netherlands in 1995 stratified by gender and presence of diabetes mellitus

\begin{tabular}{|c|c|c|c|c|}
\hline \multirow[t]{2}{*}{ Men } & \multicolumn{2}{|c|}{ Diabetic patients $(n=969)$} & \multicolumn{2}{|c|}{ Non-diabetic patients $(n=13,494)$} \\
\hline & Deaths $(n)$ & Deaths $(\%)$ & Deaths $(n)$ & Deaths $(\%)$ \\
\hline At 28 days & 170 & $17.5(15.1-19.9)$ & 1,555 & $11.5(11.0-12.1)$ \\
\hline At 1 year & 289 & $29.8(26.9-32.7)$ & 2,316 & $17.2(16.5-17.8)$ \\
\hline At 5 years & 516 & $53.3(50.1-56.4)$ & 4,116 & $30.5(29.7-31.3)$ \\
\hline \multicolumn{2}{|c|}{ Women Diabetic patients $(n=1,049)$} & \multicolumn{2}{|c|}{ Non-diabetic patients $(n=6,053)$} & \\
\hline & Deaths (n) & Deaths $(\%)$ & Deaths (n) & Deaths $(\%)$ \\
\hline At 28 days & 235 & $22.4(19.9-24.9)$ & 1,152 & $19.0(18.0-20.0)$ \\
\hline At 1 year & 368 & $35.1(32.2-38.0)$ & 1,626 & $26.9(25.7-28.0)$ \\
\hline At 5 years & 611 & $58.2(55.3-61.2)$ & 2,538 & $41.9(40.7-43.2)$ \\
\hline
\end{tabular}

Figures are numbers and percentages of deaths (95\% confidence intervals in brackets) based on actuarial life table method

5-year HR 1.53; 95\% CI 1.40-1.67). When differences in age and other covariates between diabetic and nondiabetic patients were taken into account, risk differences became nonsignificant at 28 days, but DM was still associated with a significantly higher long-term mortality in both men and women (Table 3). The interaction between gender and DM for 28-day, 1-year and 5-year mortality did not reach significance in the analyses, indicating that risks are equally elevated among men and women.

In the multivariate analyses, gender differences in mortality in nondiabetic patients varied over time with a higher mortality in women at 28 days and a higher mortality in men at 5 years (28-day HR 1.11 ; $95 \%$ CI 1.03-1.20, 5-year HR 0.93; 95\% CI 0.88-0.98). Largely comparable, yet not significant, gender differences in mortality were found in diabetic patients (28-day HR 1.09; 95\% CI 0.89-1.34, 5-year HR 0.93; 95\% CI 0.82-1.04).

\section{Discussion}

Our study provides nationwide estimates of the difference in mortality after a first hospitalized AMI between diabetic and nondiabetic patients. Long-term mortality was significantly higher in diabetic patients than in nondiabetic patients. Yet, there were no significant differences in shortterm mortality. Risks appeared to be equally elevated in men and women.

Some aspects of the study need to be addressed. In our study, the presence of DM was merely based on information 
Table 3 Multivariate analysis of the association between diabetes mellitus and short- and long-term mortality in first acute myocardial infarction patients $(n=21,565)$ by gender and age

\begin{tabular}{lllll}
\hline & Age (years) & \multicolumn{2}{l}{ Follow-up duration } & \\
\cline { 3 - 4 } & & 28 days & 1 year & 5 years \\
\hline Men & $<60$ & $1.41(0.79-2.53)$ & $1.38(0.86-2.23)$ & $1.83(1.34-2.51)$ \\
& $60-69$ & $1.46(1.02-2.08)$ & $1.73(1.31-2.28)$ & $1.80(1.46-2.21)$ \\
& $70-79$ & $1.04(0.80-1.36)$ & $1.16(0.94-1.42)$ & $1.42(1.23-1.65)$ \\
& $\geq 80$ & $1.11(0.82-1.50)$ & $1.32(1.04-1.66)$ & $1.37(1.14-1.65)$ \\
Women & All ages & $1.16(0.99-1.36)$ & $1.33(1.17-1.50)$ & $1.49(1.36-1.64)$ \\
& $<60$ & $2.06(1.09-3.88)$ & $2.16(1.25-3.75)$ & $2.13(1.40-3.25)$ \\
& $60-69$ & $1.38(0.92-2.06)$ & $1.65(1.19-2.29)$ & $1.99(1.57-2.54)$ \\
& $70-79$ & $0.94(0.72-1.22)$ & $1.12(0.92-1.37)$ & $1.38(1.19-1.60)$ \\
Total & $\geq 80$ & $1.15(0.94-1.42)$ & $1.18(0.99-1.40)$ & $1.25(1.09-1.44)$ \\
& All ages & $1.12(0.97-1.28)$ & $1.23(1.09-1.37)$ & $1.39(1.27-1.52)$ \\
& All ages & $1.13(1.02-1.26)$ & $1.27(1.17-1.38)$ & $1.44(1.35-1.53)$ \\
\hline
\end{tabular}

Figures are hazard ratios (95\% confidence intervals in brackets) with nondiabetic patients representing the reference group

Results from Cox proportional hazards analyses with diabetes mellitus, previous cardiovascular disease and ethnic origin (age- and genderspecific hazard ratios) and age (gender-specific overall hazard ratios) and gender (overall hazard ratios) included in the model

from hospital admissions and on a retrospective period of maximal 5 years. As a result, the estimate of the effect of DM is likely an overestimate due to selection of the more severe cases (those hospitalized with or due to DM), while a fairly limited number of people with less severe stages of DM (not requiring hospital care) will be diluted in the large group of people who truly did not have DM.

In our study, we were able to jointly evaluate a number of predictors of mortality using information from the hospital register. However, we were unable to examine the contribution of other predictors on our outcomes, including other risk factors, comorbidity and treatment, as registration in the hospital register is limited. Yet, this does not invalidate the finding that DM is related to a worse long-term prognosis in patients with a first AMI, as confounding is not an issue in descriptive (prognostic) research [11]. However, if cofactors such as the presence of poorly treated hypertension or dyslipidemia become the focus of investigation, the lack of more detailed information from the hospital register renders such analyses impossible.

In our study, the percentage patients with a cardiovascular history (other than AMI) was about 2 times higher in diabetic patients than in nondiabetic patients. To be sure that the worse prognosis of diabetic patients compared to nondiabetic patients was real and not merely a reflection of the difference in cardiovascular history, we repeated the analyses in diabetic and nondiabetic patients without a cardiovascular history. These analyses yielded similar findings.

The present study involved hospitalized first AMI patients. Out-of-hospital deaths from first AMI were not included. A substantial and possibly selective mortality with regard to DM might have preceded the baseline of our study, e.g. if DM were more prone to out-of-hospital death [2] then this may result in less clear differences over the rest of the first 28 days, because the early deaths were not recorded. Therefore, the study population does not represent (the diabetes distribution of) all first AMI patients in the Netherlands, but specifically hospitalized first AMI patients, and results might differ from population-based studies, which usually manage to include the out-of-hospital deaths.

As data on patients without an AMI were not available, we were not able to compare the effect of DM on mortality in patients without an AMI with the effect in patients with an AMI, i.e. whether survival after an AMI is particularly worsened beyond the poorer prognosis already conferred by the presence of DM itself.

The strength of our study lies in the validity of the registries and linkage methods, the large size and lack of selection of the cohort and the long duration of follow-up. Recently, it was shown that $99 \%$ of the personal, admission and discharge data and $84 \%$ of the principal diagnoses (validated through medical record review by medical specialists) were correctly registered in a random sample of all hospital admissions registered in the hospital register [12]. Furthermore, over $97 \%$ of the uniquely linked hospital admissions resulting from linkage of the hospital register with the population register were shown to be correctly linked [13].

Results from different studies regarding differences in mortality between diabetic and nondiabetic patients are conflicting. Consistent with our findings, it has been reported that DM has no independent predictive value on 
short-term mortality [14]. Yet, several other recent studies showed that short-term ( $<1$ year) mortality after AMI was significantly higher (approximately 1.5 to 2 times) in diabetic patients compared with nondiabetic patients [1-3, 15]. Weitzman et al. [16] found a significant higher 1-year mortality in diabetic men (odds ratio (OR) 1.5; 95\% CI 1.2-1.9), but no significant association in women. Recent studies regarding differences in long-term mortality (follow-up ranging from 4 to 10 years) after AMI showed an approximately 1.5-2.5 higher risk of dying in diabetic patients [4-8, 17]. Ishihara et al. [18] showed that DM was an independent predictor of 10-year mortality in patients with single vessel disease (OR 1.81; 95\% CI 1.27-2.54), but in patients with multivessel disease the influence of DM was nonsignificant (OR 1.17; 95\% CI 0.85-1.60). Melchior et al. [5] reported that the difference in mortality after AMI between diabetic and nondiabetic patients increased with time (relative risk ranging from 1.03 [95\% CI $0.81-1.31]$ at 30 days to $1.43[95 \% 1.24-1.66]$ at 2 months-3 years and 1.74 [95\% CI 1.36-2.23] at 79 years in patients admitted between 1990 and 1992).

We found no significant differences in short- and longterm mortality after a first AMI between men and women in diabetic patients. A few other studies examined gender differences in mortality after an AMI in diabetic patients. Crowley et al. [19] found that women with DM were at increased risk of hospital death compared to men (OR 1.37; 95\% CI 1.08-1.75), but no significant gender difference in 1-year mortality (HR 1.25; 95\% CI 0.99-1.58) and 10-year mortality (hazard ratio $1.00 ; 95 \%$ 0.99-1.58) was found. Two other studies showed a higher short-term (hospital or 28-day) mortality in women [2, 20].

The higher risk of long-term mortality in first AMI patients with DM reinforces the importance of vigorous preventive measures by lifestyle advice and drugs in these patients. Currently, achievement of lifestyle and risk factor goals for reducing mortality in diabetic patients with AMI is poor, as illustrated by Pyorala et al. [21] who reported that $20 \%$ of diabetic patients continued to smoke, $43 \%$ were obese, $57 \%$ had hypertension and $55 \%$ had hypercholesterolemia at least 6 months after hospitalization for coronary heart disease. A long-term, intensive approach consisting of behavior modification and pharmacologic therapy aimed at multiple risk factors is necessary, as it has been shown that this results in an impressive reduction in cardiovascular complications in patients with DM [22].

In conclusion, our findings in an unselected cohort covering a complete nation show that diabetic patients are at an increased risk of long-term mortality after a first acute myocardial infarction. Yet, there are no significant differences in short-term mortality. Risks appear to be equally elevated in men and women. These results stress the importance of secondary prevention by lifestyle advice and drugs in diabetic patients after a first AMI.

Acknowledgements This study was supported by a grant from the Netherlands Heart Foundation (grant number 31632501). The study was part of the project 'Cardiovascular disease in the Netherlands: figures and facts' of the Netherlands Heart Foundation. We gratefully acknowledge the members of the project's advisory committee for their helpful comments during the preparation of this paper.

\section{References}

1. Chun BY, Dobson AJ, Heller RF. The impact of diabetes on survival among patients with first myocardial infarction. Diabetes Care 1997;20:704-8.

2. Miettinen H, Lehto S, Salomaa V, Mahonen M, Niemela M, Haffner SM, Pyorala K, Tuomilehto J. Impact of diabetes on mortality after the first myocardial infarction. The FINMONICA myocardial infarction register study group. Diabetes Care 1998;21:69-75.

3. Franklin K, Goldberg RJ, Spencer F, Klein W, Budaj A, Brieger D, Marre M, Steg PG, Gowda N, Gore JM. GRACE investigators. Implications of diabetes in patients with acute coronary syndromes. The Gobal Registry of Acute Coronary Events. Arch Intern Med 2004;164:1457-63.

4. Timmer JR, OttervangerJP, Thomas K, Hoorntje JC, de Boer MJ, Suryapranata H, Zijlstra F. Zwolle myocardial infarction study group. Long-term, cause-specific mortality after myocardial infarction in diabetes. Eur Heart J 2004;25:926-31.

5. Melchior T, Kober L, Madsen CR, Seibaek M, Jensen GVH, Hildebrandt P, Torp-Pedersen C. TRACE study group. Accelerating impact of diabetes mellitus on mortality in the years following an acute myocardial infarction. Eur Heart $\mathbf{J}$ 1999;20:973-8.

6. Mukamal KJ, Nesto RW, Cohen MC, Muller JE, Maclure M, Sherwood JB, Mittleman MA. Impact of diabetes on long-term survival after acute myocardial infarction. Comparability of risk with prior myocardial infarction. Diabetes Care 2001;24:1422-7.

7. Behar S, Boyko V, Reicher-Reiss H, Goldbourt U. Ten-year survival after acute myocardial infarction: comparison of patients with and without diabetes. SPRINT study group. Secondary Prevention Reinfarction Israeli Nifedipine Trial. Am Heart J 1997;133:290-6.

8. Donnan PT, Boyle DI, Broomhall J, Hunter K, MacDonald TM, Newton RW, Morris AD. Prognosis following first acute myocardial infarction in type 2 diabetes: a comparative population study. Diabet Med 2002;19:48-55.

9. Koek HL, de Bruin A, Gast A, Gevers E, Kardaun JW, Reitsma JB, Grobbee DE, Bots ML. Decline in incidence of hospitalised acute myocardial infarction in the Netherlands from 1995 to 2000. Heart 2005;92:162-5.

10. U.S. Department of Health, Human Services. The International Statistical Classification of Diseases, Injuries and Causes of Death. Ninth Revision. Clinical Modification. Washington DC: U.S. Department of Health and Human Services, 1979.

11. Grobbee DE. Epidemiology in the right direction: the importance of descriptive research. Eur J Epidemiol 2004;19:741-4.

12. Paas GRA, Veenhuizen KCW. Research on the validity of the LMR [in Dutch]. Utrecht: Prismant 2002.

13. De Bruin A, Kardaun JWPF, Gast A, De Bruin E, Van Sijl M, Verweij G. Record linkage of hospital discharge register with population register: experiences at Statistics Netherlands. Stat J UN Econ Comm Eur 2004;21:23-32. 
14. Casella G, Savonitto S, Chiarella F, Gonzini L, Di Chiara A, Bolognese L, De Servi S, Greco C, Zonzin P, Coccolini S, Maggioni AP, Boccanelli A. BLITZ-1 Study Investigators. Clinical characteristics and outcome of diabetic patients with acute myocardial infarction. Data from the BLITZ-1 study. Ital Heart J 2005;6:374-83.

15. Mak KH, Moliterno DJ, Granger CB, Miller DP, White HD, Wilcox RG, Califf RM, Topol EJ. Influence of diabetes mellitus on clinical outcome in the thrombolytic era of acute myocardial infarction. GUSTO-I Investigators. Global Utilization of Streptokinase and Tissue Plasminogen Activator for Occluded Coronary Arteries. J Am Coll Cardiol 1997;30:171-9.

16. Weitzman S, Wang C, Rosamond WD, Chambless LE, Cooper LS, Shahar E, Goff DC. ARIC (Atherosclerosis Risk in Communities) Surveillance Study. Is diabetes an independent risk factor for mortality after myocardial infarction? The ARIC (Atherosclerosis Risk in Communities) Surveillance Study. Acta Diabetol 2004;41:77-83.

17. Lowel H, Koenig W, Engel S, Hormann A, Keil U. The impact of diabetes mellitus on survival after myocardial infarction: can it be modified by drug treatment? Results of a population-based myocardial infarction register follow-up study. Diabetologia 2000;43:218-26.
18. Ishihara M, Sato H, Kawagoe T, Shimatani Y, Kurisu S, Nishioka $\mathrm{K}$, Kouno Y, Umemura T, Nakamura S. Impact of diabetes mellitus on long term survival after acute myocardial infarction in patients with single vessel disease. Heart 2001;86:133-8.

19. Crowley A, Menon V, Lessard D, Yarzebski J, Jackson E, Gore JM, Goldberg RJ. Sex differences in survival after acute myocardial infarction in patients with diabetes mellitus (Worcester Heart Attack Study). Am Heart J 2003;146:824-31.

20. Vaccarino V, Parsons L, Every NR, Barron HV, Krumholz HM. Impact of history of diabetes mellitus on hospital mortality in men and women with first acute myocardial infarction. The National Registry of Myocardial Infarction 2 Participants. Am J Cardiol 2000;85:1486-9; A7.

21. Pyorala K, Lehto S, De Bacquer D, De Sutter J, Sans S, Keil U, Wood D, De Backer G. EUROASPIRE I Group; EUROASPIRE II Group. Risk factor management in diabetic and non-diabetic patients with coronary heart disease. Findings from the EUROASPIRE I AND II surveys. Diabetologia 2004;47:1257-65.

22. Gaede P, Vedel P, Larsen N, Jensen GV, Parving HH, Pedersen O. Multifactorial intervention and cardiovascular disease in patients with type 2 diabetes. N Engl J Med 2003;348:383-93. 